University of Nebraska - Lincoln

DigitalCommons@University of Nebraska - Lincoln

2012

\title{
Multiferroic tunnel junctions with poly(vinylidene fluoride)
}

\author{
Julian P. Velev \\ University of Nebraska-Lincoln, julian.velev@unl.edu \\ Juan M. López-Encarnación \\ University of Puerto Rico \\ John D. Burton \\ University of Nebraska-Lincoln, jburton2@unl.edu \\ Evgeny Y. Tsymbal \\ University of Nebraska-Lincoln, tsymbal@unl.edu
}

Follow this and additional works at: https://digitalcommons.unl.edu/physicstsymbal

Part of the Condensed Matter Physics Commons

Velev, Julian P.; López-Encarnación, Juan M.; Burton, John D.; and Tsymbal, Evgeny Y., "Multiferroic tunnel junctions with poly(vinylidene fluoride)" (2012). Evgeny Tsymbal Publications. 42.

https://digitalcommons.unl.edu/physicstsymbal/42

This Article is brought to you for free and open access by the Research Papers in Physics and Astronomy at DigitalCommons@University of Nebraska - Lincoln. It has been accepted for inclusion in Evgeny Tsymbal Publications by an authorized administrator of DigitalCommons@University of Nebraska - Lincoln. 


\title{
gु \\ Multiferroic tunnel junctions with poly(vinylidene fluoride)
}

\author{
Julian P. Velev, ${ }^{1,2, *}$ Juan M. López-Encarnación, ${ }^{1,3}$ J. D. Burton, ${ }^{2}$ and Evgeny Y. Tsymbal ${ }^{2}$ \\ ${ }^{1}$ Department of Physics, Institute for Functional Nanomaterials, University of Puerto Rico, San Juan, Puerto Rico 00931, USA \\ ${ }^{2}$ Department of Physics and Astronomy, Nebraska Center for Materials and Nanoscience, \\ University of Nebraska, Lincoln, Nebraska 68588, USA \\ ${ }^{3}$ Departamento de Física e Instituto de Física, Universidad Autonoma de Santo Domingo, Distrito Nacional, Dominican Republic 10105
}

(Received 12 December 2011; published 5 March 2012)

\begin{abstract}
We perform first-principles calculations based on density functional theory of the spin-resolved conductance of poly(vinylidene fluoride)- (PVDF) based multiferroic tunnel junctions (MFTJs). We consider Co/PVDF/O/Co (0001) MFTJs with one oxidized interface, representing the different experimental growth conditions for the two interfaces. We demonstrate that this natural asymmetry leads to multiple resistance states associated with different magnetization configurations of the electrodes and ferroelectric polarization orientations of the barrier. Our results indicate very high tunability of the tunneling magnetoresistance and electroresistance effects, which could be useful for logic and memory applications.
\end{abstract}

DOI: 10.1103/PhysRevB.85.125103 PACS number(s): 73.63.-b, 72.25.-b, 73.40.Gk, 73.61.Ph

\section{INTRODUCTION}

Recently, there has been a significant surge of interest in utilizing organic materials for spintronics applications. ${ }^{1,2}$ Organics are composed of light elements and exhibit very weak spin-orbit coupling, which allows the electron spin state to be preserved over long periods of time. ${ }^{3,4}$ Spin relaxation times in organic materials exceed those in inorganic materials by an order of magnitude. ${ }^{5}$ This implies spin diffusion lengths in organics of the order of hundreds of nanometers, which makes them good candidates for barriers in organic-based magnetic tunnel junctions (MTJs). Magnetic tunnel junctions exhibit a tunneling magnetoresistance (TMR) effect, which is a change in resistance of the MTJ when the relative orientation of its magnetization is altered from parallel to antiparallel. ${ }^{6}$ There have been a number of experimental investigations of spin-dependent transport in organic-based structures. ${ }^{7-14}$ The earlier experiments utilize thicker organic layers in which the electron transport is noncoherent. Dediu et al. indicated a possibility to inject spins into an organic material, sexithiophene. ${ }^{7}$ Xiong et al. demonstrated a sizable magnetoresistance effect in $\mathrm{La}_{0.67} \mathrm{Sr}_{0.33} \mathrm{MnO}_{3}(\mathrm{LSMO}) / \mathrm{Alq}_{3} / \mathrm{Co}$ spin valves where the $\mathrm{Alq}_{3}$ (tris [8-hydroxyquinoline] aluminum) was 130-250-nm thick. ${ }^{8}$ Santos et al. succeeded in achieving the tunneling regime in $\mathrm{Co} / \mathrm{Al}_{2} \mathrm{O}_{3} / \mathrm{Alq}_{3}(2 \mathrm{~nm}) / \mathrm{NiFe}$ MTJs, but the TMR was relatively modest. ${ }^{12}$ Recently, Barraud et al. reported a very large TMR effect of $300 \%$ in $\mathrm{LSMO} / \mathrm{Alq}_{3}(2 \mathrm{~nm}) / \mathrm{Co}$ MTJs. ${ }^{14}$ This large value of TMR is comparable to those found in crystalline MTJs based on $\mathrm{MgO},{ }^{15,16}$ thus indicating the feasibility of organic materials as replacements for inorganic barriers in MTJs.

Theoretical studies of the transport in organic-based MTJs are largely focused on spin-dependent transport across individual molecules (see e.g. Refs. 17 and 18 for recent reviews). It was indicated that bound states weakly coupled to the electrodes play an important role in spin-dependent transmission across molecules. Also, it was stated that the bonding between the molecule and electrodes may be engineered to a degree usually superior to that achievable in conventional inorganic heterostructures. From that point of view, transport involving extended organic systems is very promising and remains to be thoroughly investigated.

Very recently, we have investigated from first-principles organic-based MTJs based on poly(vinylidene fluoride) (PVDF) barriers. ${ }^{19}$ Poly(vinylidene fluoride) is an organic ferroelectric which exhibits robust ferroelectricity down to monolayer thickness. ${ }^{20,21}$ Using a ferroelectric thin film as a barrier in a tunnel junction adds new functionalities. First, it allows controlling the tunneling conductance in such a ferroelectric tunnel junction (FTJ) through ferroelectric polarization of the barrier. ${ }^{22}$ The associated tunneling electroresistance (TER) effect represents a resistance change with ferroelectric polarization reversal, as was predicted theoretically ${ }^{23-25}$ and confirmed experimentally. ${ }^{26-28}$ Second, using a ferroelectric barrier in an MTJ makes it multiferroic. In such a multiferroic tunnel junction (MFTJ), the TER and TMR effects coexist, as was first predicted using a simple model ${ }^{29}$ and then demonstrated by first-principles calculations. ${ }^{30,31}$ Recently, this behavior was observed experimentally in MFTJs with different ferroelectric barriers. ${ }^{32-34}$

Using the ferroelectric properties of PVDF, we have demonstrated that Co/PVDF/Fe/Co (0001) organic MFTJs exhibit multiple resistance states associated with different magnetization configurations of the electrodes and ferroelectric polarization orientations of the barrier..$^{19}$ In order to achieve these multifunctional properties, we assumed that a monolayer of $\mathrm{Fe}$ was added at one of the interfaces to make the junction asymmetric. However, the experimental growth conditions for Co/PVDF junctions could naturally lead to two very different interfaces, where one of the interfaces may be oxidized. ${ }^{35}$ This is because, in practice, the Co/PVDF interface is grown using the Langmuir-Blodgett (LB) technique, which entails depositing PVDF on the metal surface from solution. The second interface is made through evaporation of the metal on PVDF. $^{20}$ The inherent asymmetry of the deposition process suggests that the two interfaces are rather different in quality. Normally the LB-deposited interface is oxidized due to contact with air and water from the solution.

In this paper, we use first-principles density functional calculations to explore the effect of ferroelectric polarization 
on spin-dependent transport in Co/PVDF/O/Co (0001) MFTJs with asymmetric interfaces, one being clean and the other being oxidized. We demonstrate that the natural asymmetry of the interfaces leads to the coexistence of the TER and TMR effects. Moreover, we show that the TMR strongly depends on ferroelectric polarization of the barrier, and the TER is affected by the magnetization configuration of the electrodes. We argue that this high tunability of the TMR and TER effects may be useful for logic applications.

\section{COMPUTATIONAL METHODS AND STRUCTURAL MODEL}

We calculate the electronic structure of $\mathrm{Co} / \mathrm{PVDF} / \mathrm{O} / \mathrm{Co}$ (0001) MFTJs using first-principles calculations based on density functional theory (DFT) implemented in a plane-wave pseudopotential method within the Quantum-Espresso (QE) package. ${ }^{36}$ The Perdew-Burke-Ernzerhof (PBE) exchangecorrelation functional with energy cutoff of $400 \mathrm{eV}$ for the plane-wave expansion and a $4 \times 12 \times 1$ Monkhorst-Pack grid for $k$-point sampling are used in the calculations. Then conductance is calculated using a wave function-matching formalism implemented for plane waves and pseudopotentials in the QE package. ${ }^{37,38}$ The structure in Fig. 1 is considered as the scattering region, ideally attached on both sides to semiinfinite hcp Co (0001) leads. The transmission and reflection matrices are then obtained by matching the wave functions in the scattering region to appropriate linear combinations of the Bloch states in the left and right leads. The conductance calculations are performed at zero bias using a uniform $30 \times$ $100 k$-point mesh in the two-dimensional Brillouin zone.

Figure 1 shows the structural model for a $\mathrm{Co} / \mathrm{PVDF} / \mathrm{O} / \mathrm{Co}$ MFTJ, where we assume an $\mathrm{O}$ monolayer present at the right interface. We considered several possible $\mathrm{O}$ adsorption sites on the Co surface. The most energetically favorable site is the threefold hcp-hollow site in which the $\mathrm{O}$ atom is about $1.04 \AA$ above the surface between three Co atoms and on top of the $\mathrm{Co}$ atom in the monolayer below, as illustrated in Fig. 2. The $\mathrm{O}$ fcc-hollow site is found to be very close in energy $(+20 \mathrm{meV})$ and at the same distance (Fig. 2). The slightly stronger bonding in the hcp site is probably due to the presence of the second Co

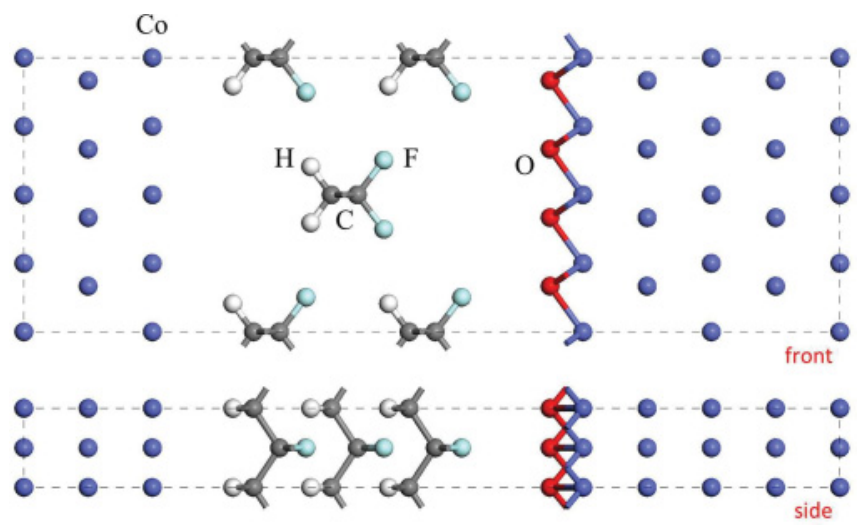

FIG. 1. (Color online) Atomic structure of $\mathrm{Co} / \mathrm{PVDF} / \mathrm{O} / \mathrm{Co}$ (0001) MFTJs with three monolayers of PVDF. The right interface contains a monolayer of oxygen. Ferroelectric polarization is pointing to the left.

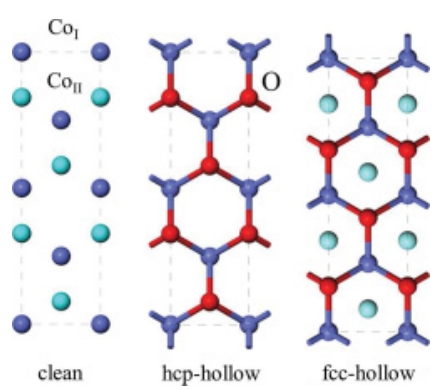

FIG. 2. (Color online) Atomic structure of clean and oxidized Co surface. $\mathrm{O}$ atoms (red/dark gray) are in either the hcp- or fcc-hollow sites. Blue (medium gray) $\mathrm{Co}$ atoms $\left(\mathrm{Co}_{\mathrm{I}}\right)$ are at the surface and light blue (light gray) $\mathrm{Co}$ atoms $\left(\mathrm{CO}_{\text {II }}\right)$ are at the layer immediately below the surface.

atom below the surface. The $\mathrm{O}$ bridge and atop adsorption sites are found to be much higher in energy. This is consistent with previous studies. ${ }^{39,40}$ Due to the small difference in energy and structure between the hcp- and fcc-hollow sites, we consider both of them in our study.

Next, we consider the PVDF barrier. The atomic and electronic structure of bulk PVDF $\left[-\left(\mathrm{CH}_{2}-\mathrm{CF}_{2}\right)_{n}-\right]$ is well understood. ${ }^{19,21}$ The theoretical band gap of $5.8 \mathrm{eV}$ is in good agreement with experiment $(6.5 \mathrm{eV}) .{ }^{41}$ The spontaneous electric polarization is $19.3 \mu \mathrm{C} / \mathrm{cm}^{2}$ pointing from $\mathrm{F}(-)$ to $\mathrm{H}(+)$ atoms, and the reversal of ferroelectric polarization is associated with the rotation of the $\mathrm{CH}_{2}-\mathrm{CF}_{2}$ complex so that the $\mathrm{H}$ and $\mathrm{F}$ atoms are interchanged. We use the simplified unit cell for PVDF, which ignores the small dihedral deflections between the monomers. ${ }^{42}$

The hcp Co $4 \times 1$ supercell $(a=8.685 \AA, b=2.507 \AA)$ matches within $2 \%$ with the PVDF unit cell $(a=8.58, b=$ 5.12, $c=4.91 \AA$ ), where we choose the $\mathrm{C}$ chains to be atop of rows of Co. As before, we find that PVDF is physisorbed on the surface of Co. ${ }^{19}$ Density functional theory does not predict the weak bonding correctly; therefore, in order to determine the interface separation, we use dispersion-corrected DFT (DFT-D), in which the van der Waals interactions are included semi-empirically. ${ }^{43}$ The equilibrium interface distances for the clean Co interface are 2.55 and $3.04 \AA(\mathrm{Co}: \mathrm{H}$ and $\mathrm{Co}: \mathrm{F}$ interfaces, respectively). The equilibrium interface separations at the oxidized interface are 2.31 and $2.80 \AA$ (CoO: $\mathrm{H}$ and $\mathrm{CoO}: \mathrm{F}$ interfaces, respectively, where the distance is measured from the $\mathrm{O}$ ).

\section{ELECTRONIC STRUCTURE}

After determining the atomic structure of the Co/PVDF/ $\mathrm{O} / \mathrm{Co} \mathrm{MFTJ}$, we turn to the study of the electronic structure. Figure 3 shows the calculated local density of states (DOS) in the $\mathrm{Co} / \mathrm{PVDF} / \mathrm{O} / \mathrm{Co} \mathrm{MFTJ}$ for $\mathrm{O}$ in the hcp-hollow site. We find that the DOS of Co at the clean interface [Fig. 3(a)] is very similar to the DOS of bulk Co (not shown). The latter is characterized by the large exchange splitting of the Co $d$ band, resulting in fully occupied majority-spin $3 d$ states and partially occupied minority-spin $3 d$ states. The magnetic moment of the surface Co, $1.79 \mu_{B}$, is also similar to that of bulk Co, $1.70 \mu_{B}$. The local DOS of Co at the oxidized interface [Fig. 3(e)] is, however, very different from that in 


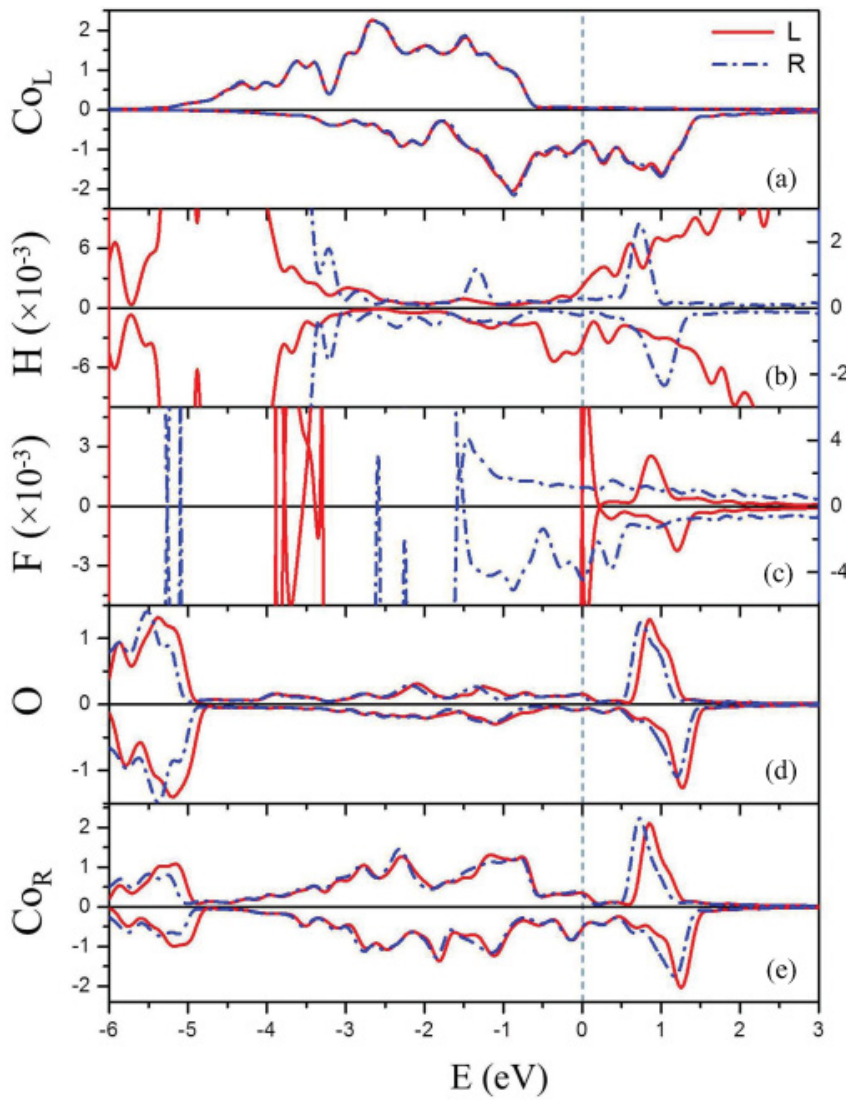

FIG. 3. (Color online) Spin-polarized local density of states (DOS) at the interfaces of Co/PVDF/O/Co MFTJ. Local DOS for (a) Co at clean (left) interface and (e) Co at oxidized (right) interface. Local DOS on (b) $\mathrm{H}$ and (c) $\mathrm{F}$ atoms adjacent to the interface, and on (d) O atoms. Top (bottom) panels show majority (minority)-spin DOS. Blue (medium gray) dashed and red (dark gray) solid lines correspond to polarization to the left and right, respectively. DOS on $\mathrm{H}$ and $\mathrm{F}$ are multiplied by $10^{3}$ and plotted on a different scale (red/dark gray on the left axis and blue/medium gray on the right axis).

the bulk. The $\mathrm{O}$ monolayer is chemically bonded to the Co surface, as is evident from the bonding and antibonding states situated around $-5 \mathrm{eV}$ below and $1 \mathrm{eV}$ above the Fermi level, respectively [Figs. 3(d) and 3(e)]. This bonding almost completely quenches the magnetism of the interfacial Co layer. We find that the exchange splitting of the Co $d$ band becomes small (about $0.4 \mathrm{eV}$, as seen from the spin splitting of the antibonding states), and the magnetic moment is reduced down to $0.30 \mu_{B}$. These findings are consistent with the results obtained previously for the oxidized Co surface. ${ }^{39}$

We find that the reversal of the PVDF polarization direction has little effect on the Co DOS at the clean interface [red and blue lines in Fig. 3(a)]. However, at the oxidized interface, we see a rigid shift of the antibonding state by about $0.1 \mathrm{eV}$ with switching polarization [red and blue lines in Figs. 3(d) and $3(\mathrm{e})]$. This shift is due to the screening of the polarization charge at the interfaces. Electrons are removed from the interface (bands shifted up) when the negative $\mathrm{F}$ points towards the interface (polarization left), and attracted to the interface (shifted down) when the positive $\mathrm{H}$ points towards the interface (polarization right). The effect of the screening is more pronounced at the oxidized interface because of the smaller DOS. The majority-spin antibonding state lies closer to the Fermi energy, and thus this shift affects the majority-spin DOS at the Fermi level stronger than the minority-spin DOS.

By comparing deep corelike PVDF levels in the bulk and in the MFTJ, we find that the Fermi energy is located just about midgap of PVDF. The induced DOS on the $\mathrm{H}$ and $\mathrm{F}$ atoms adjacent to the clean interface [red line in Fig. 3(b) for $\mathrm{H}$ and blue line in Fig. 3(c) for F] are the same as we calculated previously for the Co/PVDF/Co MFTJ ${ }^{19}$ except the position of the Fermi level, which in this case lies a couple of tenths of $\mathrm{eV}$ lower. The DOS on the $\mathrm{H}$ and $\mathrm{F}$ atoms adjacent to the oxidized interface [blue line in Fig. 3(b) for $\mathrm{H}$ and red line in Fig. 3(c) for F] are pronouncedly different. The induced DOS due to the antibonding state is clearly visible on both $\mathrm{H}$ and $\mathrm{F}$. The induced DOS on the $\mathrm{H}$ atom at the oxidized interface is more than an order of magnitude smaller than that at the clean interface. This is due to the smaller DOS of the oxidized interface itself, in addition to the larger interface separation between the PVDF and the Co at this interface. The induced DOS on the F atom at the oxidized interface is also smaller for the same reasons. However, we find that the F- $p$ band is now pulled all the way to the Fermi level due to the F-O electrostatic interaction.

The electronic structure of the junction with $\mathrm{O}$ in the fcc-hollow site is quite similar. Figure 4 compares the DOS for the two cases. The main difference is the position of the $\mathrm{Co}-\mathrm{O}$ antibonding levels, which are higher in energy for the fcc structure by about $0.15 \mathrm{eV}$ due to the weaker bonding [Fig. 4(c)]. The main effect is the pulling of the F-p band higher in energy [Fig. 4(b)], which substantially changes the

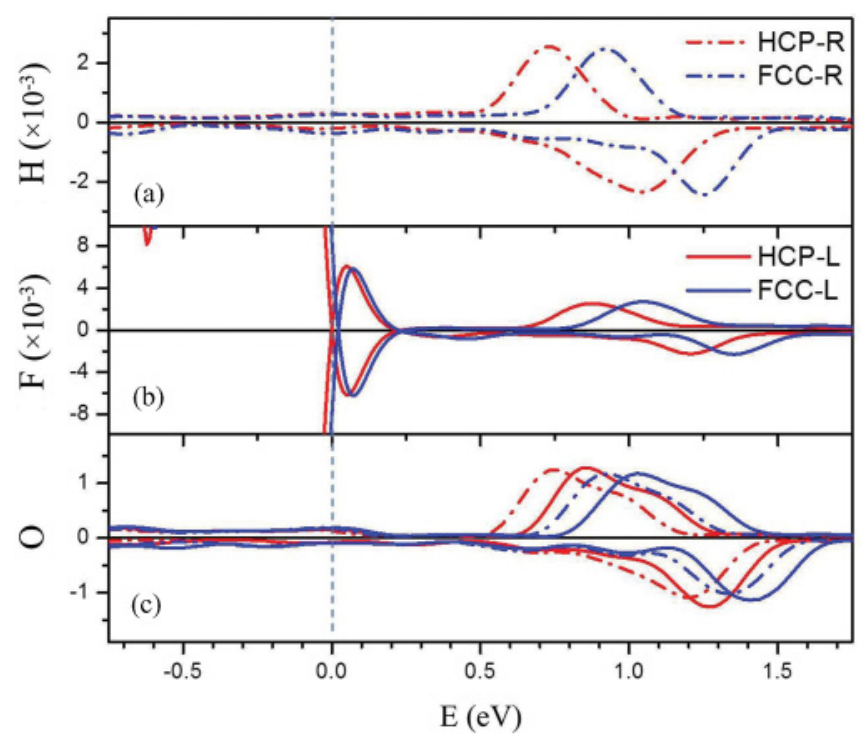

FIG. 4. (Color online) Comparison of the density of states (DOS) at the interface of the $\mathrm{Co} / \mathrm{PVDF} / \mathrm{O} / \mathrm{Co}$ MFTJ for the hcp- and fccadsorption sites. Induced DOS on (a) $\mathrm{H}$ and (b) F atoms at the oxidized interface; and (c) DOS on the $\mathrm{O}$ atom at the interface. Red/dark gray (blue/medium gray) lines show results for $\mathrm{O}$ in hcp (fcc) sites. Solid (dashed-dotted) lines indicate polarization pointing left (right). Induced DOS on $\mathrm{H}$ and $\mathrm{F}$ atoms is multiplied by $10^{3}$. 
DOS at the Fermi level and is expected to have an effect on the conductance.

\section{CONDUCTANCE, TMR, AND TER}

Finally, we use the first-principles electronic structure to calculate the conductance of the Co/PVDF/O/Co MFTJ. The conductance is obtained for parallel $(P)$ and antiparallel $(A P)$ magnetization orientation of the electrodes and for ferroelectric polarization in the barrier pointing to the left or to the right. The results are shown in Table I, where also the conductance of Co/PVDF/Co MTJ with clean interfaces is given for comparison. The TMR is defined as $\mathrm{TMR}=$ $\left(G_{P}-G_{A P}\right) /\left(G_{P}+G_{A P}\right)$, where $G_{P}=G_{\uparrow \uparrow}+G_{\downarrow \downarrow}$ is the conductance for the $P$ configuration, $G_{A P}=G_{\uparrow \downarrow}+G_{\downarrow \uparrow}$ is the conductance for the $A P$ configuration, and vertical arrows indicate the spin direction. The conductance for the $A P$ configuration is calculated by doubling the unit cell in the current direction and setting the magnetization in the second Co slab opposite to the first. The TER ratio is defined as TER $=\left(G_{\leftarrow}-G_{\rightarrow}\right) /\left(G_{\leftarrow}+G_{\rightarrow}\right)$, where $G_{\leftarrow}$ is the conductance for the PVDF polarization pointing to the left, $G_{\rightarrow}$ the conductance for the PVDF polarization pointing to the right, and horizontal arrows indicate the polarization direction.

The data in Table I show that the Co/PVDF/O/Co MFTJ displays four distinct resistance states due to the coexistence of TMR and TER effects. The conductance in all configurations of the oxidized MFTJ is about two orders of magnitude less than that for the MFTJ with clean interfaces. This is due to the larger barrier thickness for the MFTJ with the oxidized interface and the smaller DOS on the oxidized Co. We find that the spin polarization of the conductance and the TMR effect are substantial for both structural models and either ferroelectric polarization orientation.

Consistent with the previous studies, interfaces play a decisive role in controlling TMR. ${ }^{40}$ The basic mechanism of TMR can be interpreted in the lines of the effect of oxidation

TABLE I. Conductance per unit cell area, TMR and TER for $\mathrm{Co} / \mathrm{PVDF} / \mathrm{O} / \mathrm{Co}$ (0001) MFTJ. Majority- and minority-spin conductance for parallel magnetization of the electrodes $(\uparrow \uparrow$ and $\downarrow \downarrow$, respectively) and the total conductance for the antiparallel magnetization $(\uparrow \downarrow+\downarrow \uparrow)$ are shown for the PVDF polarization oriented left $(\leftarrow)$ and right $(\rightarrow)$.

\begin{tabular}{|c|c|c|c|c|}
\hline & \multicolumn{3}{|c|}{$\mathrm{G}\left(10^{-7} \mathrm{e}^{2} / h\right)$} & \multirow{2}{*}{$\begin{array}{c}\text { TMR } \\
(\%)\end{array}$} \\
\hline & $\uparrow \uparrow$ & $\downarrow \downarrow$ & $\uparrow \downarrow+\downarrow \uparrow$ & \\
\hline \multicolumn{5}{|c|}{$\mathrm{Co} / \mathrm{PVDF} / \mathrm{Co}$} \\
\hline$\leftarrow(\rightarrow)$ & 104.59 & 235.56 & 471.23 & -16.3 \\
\hline \multicolumn{5}{|c|}{$\mathrm{Co} / \mathrm{PVDF} / \mathrm{O}(\mathrm{hcp}) / \mathrm{Co}$} \\
\hline$\leftarrow$ & 4.40 & 2.54 & 7.64 & -4.6 \\
\hline$\rightarrow$ & 2.74 & 2.11 & 10.04 & -34.8 \\
\hline TER $(\%)$ & & 17.7 & -13.7 & \\
\hline \multicolumn{5}{|c|}{$\mathrm{Co} / \mathrm{PVDF} / \mathrm{O}(\mathrm{fcc}) / \mathrm{Co}$} \\
\hline$\leftarrow$ & 4.42 & 8.70 & 11.1 & 8.7 \\
\hline$\rightarrow$ & 2.43 & 6.21 & 12.33 & -17.0 \\
\hline TER $(\%)$ & & 20.0 & -5.6 & \\
\hline
\end{tabular}

on the Co surface. ${ }^{39}$ The spin polarization of the clean Co interface is negative due to the partially filled minority $d$ states. Oxidation creates a strong covalent bond between the $\mathrm{O}$ and the $\mathrm{Co}$ at the interface. The $\mathrm{Co}-\mathrm{O}$ bonding state is below the Co- $d$ bands and does not affect the conductance. However, the Co-O antibonding state has a large peak around $1 \mathrm{eV}$ above the Fermi level and a wide portion which coincides in energy with the bulk Co- $d$ bands. The net effect is that the spin polarization of the oxidized interface becomes positive. Since TMR can be expressed through the interface spin polarizations as TMR $=P_{L} P_{R}$, where $P_{L}\left(P_{R}\right)$ the spin polarization of the left (right) interface, this leads to inverse TMR. ${ }^{44}$

We find that the ferroelectric polarization of PVDF has a dramatic effect on TMR. In the case of the hcp adsorption site, TMR changes from about $5 \%$ to about $35 \%$ with reversal of ferroelectric polarization. In the case of the fcc adsorption site, the TMR changes sign. In order to explain this tunability of TMR, we resort to the model developed in our previous paper. ${ }^{19}$ The transport spin polarization of the interface can be expressed as $P=\left(T_{\uparrow}-T_{\downarrow}\right) /\left(T_{\uparrow}+T_{\downarrow}\right)$, where $T$ is the average spin-dependent interface transmission function (ITF) across the interface. ${ }^{39}$ The ITF can be interpreted in terms of the electrode-induced DOS inside the barrier. ${ }^{4,46}$ Thus, the ITFs for the various interfaces can be obtained from the DOS in Fig. 3. In the case of the hcp-hollow site, we observe that, for the clean interface $T_{\mathrm{Co}: \mathrm{H}}^{\uparrow}<T_{\mathrm{Co}: \mathrm{H}}^{\downarrow}$ and $T_{\mathrm{Co}: \mathrm{F}}^{\uparrow}<T_{\mathrm{Co}: \mathrm{F}}^{\downarrow}$, which means that the spin polarization of both interfaces is negative $P_{\mathrm{Co}: \mathrm{H}}<0$ and $P_{\mathrm{Co}: \mathrm{F}}<0$. Judging the induced DOS at the oxidized interface is much more difficult because the DOS is too small, and the differences are within the precision of the calculation. However, TMR is consistent with the fact that $P_{\mathrm{Co}: \mathrm{H}}>0$ and $P_{\mathrm{Co}: \mathrm{F}}>0$. Thus, we obtain inverse $\mathrm{TMR}$ in both cases $\mathrm{TMR} \leftarrow=P_{\mathrm{Co}: \mathrm{H}} P_{\mathrm{CoO}: \mathrm{F}}<0$ and $\mathrm{TMR}_{\rightarrow}=$ $P_{\mathrm{Co}: \mathrm{F}} P_{\mathrm{CoO}: \mathrm{H}}<0$. At the same time, for $\mathrm{O}$ in the fcc-hollow site, the main difference is the position of the antibonding state further up in energy, which pulls the F- $p$ band closer to the Fermi level. This shift is consistent with the change of the spin polarization at the F:O interface to negative $P_{\mathrm{CoO}: \mathrm{F}}<0$ and the change of TMR to positive $\mathrm{TMR}_{\leftarrow}=P_{\mathrm{Co}: \mathrm{H}} P_{\mathrm{CoO}: \mathrm{F}}>0$ for ferroelectric polarization pointing to the left. For ferroelectric polarization pointing to the right, the TMR remains unchanged and inverse. The slightly larger DOS on the interface $F$ atom is also consistent with the enhanced conductance both for $P$ and $A P$ configurations in the fcc-hollow case.

In addition to the robust TMR, we find a sizable TER effect. This is due to the natural structural asymmetry created by the growth conditions. This structural difference between the interfaces translates into a large difference in the electronic structure. At the clean metal interface, screening plays an insignificant role, while at the oxidized interface, it leads to substantial changes in the electronic structure, the most pronounced of which is the moving of the F- $p$ band close to the Fermi level. In the $P$ configuration for both structural models $G_{\leftarrow}^{P}=T_{\mathrm{Co}: \mathrm{H}}^{\uparrow} T_{\mathrm{CoO}: \mathrm{F}}^{\uparrow}+T_{\mathrm{Co}: \mathrm{H}}^{\downarrow} T_{\mathrm{CoO}: \mathrm{F}}^{\downarrow}$ and $G_{\rightarrow}^{P}=$ $T_{\mathrm{Co}: \mathrm{F}}^{\uparrow} T_{\mathrm{CoO}: \mathrm{H}}^{\uparrow}+T_{\mathrm{Co}: \mathrm{F}}^{\downarrow} T_{\mathrm{CoO}: \mathrm{H}}^{\downarrow}$. It is expected that $G_{\leftarrow}^{P}>G_{\rightarrow}^{P}$ because $T_{\mathrm{CoO}: \mathrm{H}} \ll T_{\mathrm{Co}: \mathrm{H}}$ while $T_{\mathrm{CoO}: \mathrm{F}}<T_{\mathrm{Co}: \mathrm{F}}$, and therefore TER $>0$. Indeed, we see in Table I that the spin polarization of conductance in both spin channels increases when the ferroelectric polarization switches to the left. In addition, there 
is an effect resulting from the spin polarization of the DOS. Since in the hcp-hollow case, the spin polarizations of the two interfaces are opposite, there is a spin mismatch effect where, in both spin channels, the tunneling occurs between high and low DOS at the interfaces. In the fcc-hollow case, when the ferroelectric polarization is oriented to the left, the spin polarization is the same at both interfaces, which leads to larger conductance and thus larger TER. In the $A P$ configuration for both structural models, $G_{\leftarrow}^{A P}=T_{\mathrm{Co}: \mathrm{H}}^{\uparrow} T_{\mathrm{CoO}: \mathrm{F}}^{\downarrow}+T_{\mathrm{Co}: \mathrm{H}}^{\downarrow} T_{\mathrm{CoO}: \mathrm{F}}^{\uparrow}$ and $G_{\rightarrow}^{A P}=T_{\mathrm{Co}: \mathrm{F}}^{\uparrow} T_{\mathrm{CoO}: \mathrm{H}}^{\downarrow}+T_{\mathrm{Co:F}}^{\downarrow} T_{\mathrm{CoO}: \mathrm{H}}^{\uparrow}$. Now the effect of the spin polarized DOS is reversed. In all configurations with the opposite spin polarization at the interfaces, the $A P$ configuration couples low DOS at one interface to low DOS at the other interface in one spin channel and high DOS to high DOS in the other spin channel. This leads to a sizable increase in the conductance in one of the spin channels. Thus, the TER effect changes sign so that TER $<0$. However, this behavior is opposite in the fcc-hollow site structure when the ferroelectric polarization is pointed to the left. In this case, the TER effect is smaller in the $A P$ configuration than in the $P$ configuration.

\section{CONCLUSIONS}

We have performed density functional calculations of the spin-resolved conductance of $\mathrm{Co} / \mathrm{PVDF} / \mathrm{O} / \mathrm{Co}$ (0001) MFTJs with asymmetric interfaces, one being clean and the other oxidized. We find that, while the clean interface has high DOS with negative spin polarization, the oxidized interface has low DOS with positive spin polarization. Switching the ferroelectric polarization of PVDF shifts the Co-O antibonding state at the oxidized interface by about $0.2 \mathrm{eV}$ due to screening of the polarization charges. This alters the electronic properties of the interface (in particular the position of the F- $p$ band) and affects the interface transmission properties. As a result, we obtain very high sensitivity of the conductance to the ferroelectric polarization direction and highly tunable TER and TMR effects. The main difference between the oxygen hcp and fcc adsorption sites is in the position of the antibonding states with these being slightly higher in energy in the latter case.

Overall, we find that employing ferroelectric organic thin films as tunnel barriers in magnetic tunnel junctions could be potentially very interesting for spintronics applications. We predict that, not only do the TMR and TER effects exist in organic MFTJs, but they can be highly tunable. The natural asymmetry in the growth conditions of the PVDF-based MFTJs leads to the desired TER effect without any special interface engineering. Moreover, in such organic MFTJs, the TER can change sign in applied magnetic field, and the TMR magnitude can be significantly altered by electric field, making these MFTJs interesting for hybrid memory-logic devices.

\section{ACKNOWLEDGMENTS}

This work was supported by the National Science Foundation at the University of Puerto Rico (Grant Nos. DMR1105474 and EPS-1010094) and at the University of NebraskaLincoln (Grant Nos. DMR-0820521 and EPS-1010674). Computations were performed at the University of Nebraska Holland Computing Center. *jvelev@gmail.com

${ }^{1}$ V. A. Dediu, L. E. Hueso, I. Bergenti, and C. Taliani, Nat. Mater. 8, 707 (2009).

${ }^{2}$ G. Szulczewski, S. Sanvito, and J. M. D. Coey, Nat. Mater. 8, 693 (2009).

${ }^{3}$ A. J. Drew, J. Hoppler, L. Schulz, F. L. Pratt, P. Desai, P. Shakya, T. Kreouzis, W. P. Gillin, A. Suter, N. A. Morley, V. K. Malik, A. Dubroka, K. W. Kim, H. Bouyanfif, F. Bourqui, C. Bernhard, R. Scheuermann, G. J. Nieuwenhuys, T. Prokscha, and E. Morenzoni, Nat. Mater. 8, 109 (2009).

${ }^{4}$ M. Cinchetti, K. Heimer, J. P. Wüstenberg, O. Andreyev, M. Bauer, S. Lach, C. Ziegler, Y. Gao, and M. Aeschlimann, Nat. Mater. 8, 115 (2009).

${ }^{5}$ I. Zutic, J. Fabian, and S. Das Sarma, Rev. Mod. Phys. 76, 323 (2004).

${ }^{6}$ E. Y. Tsymbal, O. N. Mryasov, and P. R. LeClair, J. Phys.: Condens. Matter 15, R109 (2003).

${ }^{7}$ V. Dediu, M. Murgia, F. C. Matacotta, C. Taliani, and S. Barbanera, Solid State Commun. 122, 181 (2002).

${ }^{8}$ Z. H. Xiong, D. Wu, Z. V. Vardeny, and J. Shi, Nature 427, 821 (2004).

${ }^{9}$ F. J. Wang, C. G. Yang, Z. V. Vardeny, and X. G. Li, Phys. Rev. B 75, 245324 (2007).

${ }^{10}$ V. Dediu, L. E. Hueso, I. Bergenti, A. Riminucci, F. Borgatti, P. Graziosi, C. Newby, F. Casoli, M. P. De Jong, C. Taliani, and Y. Zhan, Phys. Rev. B 78, 115203 (2008).
${ }^{11}$ D. Sun, L. Yin, C. Sun, H. Guo, Z. Gai, X. G. Zhang, T. Z. Ward,

Z. Cheng, and J. Shen, Phys. Rev. Lett. 104, 236602 (2010).

${ }^{12}$ T. S. Santos, J. S. Lee, P. Migdal, I. C. Lekshmi, B. Satpati, and J. S. Moodera, Phys. Rev. Lett. 98, 016601 (2007).

${ }^{13}$ J. W. Yoo, C. Y. Chen, H. W. Jang, C. W. Bark, V. N. Prigodin,

C. B. Eom, and A. J. Epstein, Nat. Mater. 9, 638 (2010).

${ }^{14}$ C. Barraud, P. Seneor, R. Mattana, S. Fusil, K. Bouzehouane, C. Deranlot, P. Graziosi, L. Hueso, I. Bergenti, V. A. Dediu, F. Petroff, and A. Fert, Nature Phys. 6, 615 (2010).

${ }^{15}$ S. S. P. Parkin, C. Kaiser, A. Panchula, P. M. Rice, and B. Hughes, Nat. Mater. 3, 862 (2004).

${ }^{16}$ S. Yuasa, T. Nagahama, A. Fukushima, Y. Suzuki, and K. Ando, Nat. Mater. 3, 868 (2004).

${ }^{17}$ S. Sanvito, in Handbook of Spin Transport and Magnetism, edited by E. Y. Tsymbal and I. Žutić, Chapter 33 (Chapman and Hall/CRC, Taylor \& Francis Group, 2011), pp. 649-661.

${ }^{18}$ S. Sanvito and A. R. Rocha, J. Comp. Theor. Nanosci. 3, 624 (2006).

${ }^{19}$ J. M. López, J. D. Burton, E. Y. Tsymbal, and J. P. Velev, Nano Lett. 11, 599 (2011).

${ }^{20}$ A. V. Bune, V. M. Fridkin, S. Ducharme, L. M. Blinov, S. P. Palto, A. Sorokin, S. G. Yudin, and A. Zlatkin, Nature 391, 874 (1998).

${ }^{21}$ S. M. Nakhmanson, M. B. Buongiorno Nardelli, and J. Bernholc, Phys. Rev. B 72, 115210 (2005).

${ }^{22}$ E. Y. Tsymbal and H. Kohlstedt, Science 313, 181 (2006). 
${ }^{23}$ M. Y. Zhuravlev, R. F. Sabirianov, S. S. Jaswal, and E. Y. Tsymbal, Phys. Rev. Lett. 94, 246802 (2005).

${ }^{24}$ H. Kohlstedt, N. A. Pertsev, J. Rodriguez Contreras, and R. Waser, Phys. Rev. B 72, 125341 (2005).

${ }^{25}$ J. P. Velev, C. G. Duan, K. D. Belashchenko, S. S. Jaswal, and E. Y. Tsymbal, Phys. Rev. Lett. 98, 137201 (2007).

${ }^{26}$ V. Garcia, S. Fusil, K. Bouzehouane, S. Enouz-Vedrenne, N. D. Mathur, A. Barthélémy, and M. Bibes, Nature 460, 81 (2009).

${ }^{27}$ P. Maksymovych, S. Jesse, P. Yu, R. Ramesh, A. P. Baddorf, and S. V. Kalinin, Science 324, 1421 (2009).

${ }^{28}$ A. Gruverman, D. Wu, H. Lu, Y. Wang, H. W. Jang, C. M. Folkman, M. Y. Zhuravlev, D. Felker, M. Rzchowski, C. B. Eom, and E. Y. Tsymbal, Nano Lett. 9, 3539 (2009).

${ }^{29}$ M. Y. Zhuravlev, S. S. Jaswal, E. Y. Tsymbal, and R. F. Sabirianov, Appl. Phys. Lett. 87, 222114 (2005).

${ }^{30}$ J. P. Velev, C. G. Duan, J. D. Burton, A. Smogunov, M. K. Niranjan, E. Tosatti, S. S. Jaswal, and E. Y. Tsymbal, Nano Lett. 9, 427 (2009).

${ }^{31}$ J. D. Burton and E. Y. Tsymbal, Phys. Rev. Lett. 106, 157203 (2011).

${ }^{32}$ V. Garcia, M. Bibes, L. Bocher, S. Valencia, F. Kronast, A. Crassous, X. Moya, S. Enouz-Vedrenne, A. Gloter, D. Imhoff, C. Deranlot, N. D. Mathur, S. Fusil, K. Bouzehouane, and A. Barthelemy, Science 327, 1106 (2010).

${ }^{33}$ M. Hambe, A. Petraru, N. A. Pertsev, P. Munroe, V. Nagarajan, and H. Kohlstedt, Adv. Funct. Mater. 20, 2436 (2010).

${ }^{34}$ S. Valencia, A. Crassous, L. Bocher, V. Garcia, X. Moya, R. O. Cherifi, C. Deranlot, K. Bouzehouane, S. Fusil, A. Zobelli, A. Gloter, N. D. Mathur, A. Gaupp, R. Abrudan, F. Radu, A. Barthélémy, and M. Bibes, Nat. Mater. 10, 753 (2011).

${ }^{35}$ A. Mardana, S. Ducharme, and S. Adenwalla, Nano Lett. 11, 3862 (2011).

${ }^{36}$ P. Giannozzi, S. Baroni, N. Bonini, M. Calandra, R. Car, C. Cavazzoni, D. Ceresoli, G. L. Chiarotti, M. Cococcioni, I. Dabo, A. Dal Corso, S. de Gironcoli, S. Fabris, G. Fratesi, R. Gebauer, U. Gerstmann, C. Gougoussis, A. Kokalj, M. Lazzeri, L. Martin-
Samos, N. Marzari, F. Mauri, R. Mazzarello, S. Paolini, A. Pasquarello, L. Paulatto, C. Sbraccia, S. Scandolo, G. Sclauzero, A. P. Seitsonen, A. Smogunov, P. Umari, and R. M. Wentzcovitch, J. Phys.:Condens. Matter 21, 395502 (2009); see [http://www.quantum-espresso.org].

${ }^{37}$ H. J. Choi and J. Ihm, Phys. Rev. B 59, 2267 (1999).

${ }^{38}$ A. Smogunov, A. Dal Corso, and E. Tosatti, Phys. Rev. B 70, 045417 (2004).

${ }^{39}$ K. D. Belashchenko, E. Y. Tsymbal, M. van Schilfgaarde, D. A. Stewart, I. I. Oleinik, and S. S. Jaswal, Phys. Rev. B 69, 174408 (2004).

${ }^{40}$ E. Y. Tsymbal, K. D. Belashchenko, J. P. Velev, S. S. Jaswal, M. van Schilfgaarde, I. I. Oleynik, and D. A. Stewart, Prog. Mat. Sci. 52, 401 (2007).

${ }^{41}$ J. Choi, P. A. Dowben, S. Pebley, A. V. Bune, S. Ducharme, V. M. Fridkin, S. P. Palto, and N. Petukhova, Phys. Rev. Lett. 80, 1328 (1998).

${ }^{42}$ R. Hasegawa, Y. Takahashi, Y. Chatani, and H. Tadokoro, Polymer Journal 3, 600 (1971).

${ }^{43}$ V. Barone, M. Casarin, D. Forrer, M. Pavone, M. Sambi, and A. Vittadini, J. Comput. Chem. 30, 934 (2009).

${ }^{44} \mathrm{We}$ note the relevant oxygen-induced change in the spinpolarization sign at the oxidized Fe (001) surface predicted theoretically: E. Y. Tsymbal, I. I. Oleinik, and D. G. Pettifor, J. Appl. Phys. 87, 5230 (2000). We also note the related inversion of TMR due to the negative spin polarization at the $\mathrm{Co} / \mathrm{SrTiO} 3$ interface observed experimentally: J. M. De Teresa, A. Barthélémy, A. Fert, J. P. Contour, F. Montaigne, and P. Seneor, 286, 507 (1999); and explained theoretically: J. P. Velev, K. D. Belashchenko, D. A. Stewart, M. van Schilfgaarde, S. S. Jaswal, and E. Y. Tsymbal, Phys. Rev. Lett. 95, 216601 (2005).

${ }^{45}$ K. D. Belashchenko and E. Y. Tsymbal, in Handbook of Spin Transport and Magnetism, edited by E. Y. Tsymbal and I. Žutić, Chapter 12 (Chapman and Hall/CRC, Taylor \& Francis Group, 2011), pp. 233-250.

${ }^{46}$ J. Tersoff and D. R. Hamann, Phys. Rev. B 31, 805 (1985). 